\title{
Benefits and limitations of imaging multiples: Mirror migration
}

\author{
Sherif M. Hanafy ${ }^{1}$, Yunsong Huang ${ }^{2}$, and Gerard T. Schuster ${ }^{1}$
}

Note: This is the first of two articles in this special section that explore the benefits and limitations of imaging multiples. Also see Guo et al., this issue.

\section{Abstract}

The benefits and limitations of imaging multiples are reviewed for mirror migration. Synthetic and field data examples are used to characterize the effectiveness of migrating multiples relative to primary imaging.

\section{Introduction}

One of humankind's earliest applications of multiple imaging appeared more than 2 million years ago when the first hominids gazed into a pool of water at Olduvai Gorge, Tanzania. Those reflected images, focused through their eyes, might have initiated our earliest musings of self-awareness.

The focusing of light multiples to the image plane of our eye is nature's natural migration operator. This is because focusing by an optical lens is mathematically equivalent to the seismic-focusing operation of a migration operator (Figures 1a and 1b) (Schuster, 2001). Therefore, we can say that multiples have been migrated since the dawn of mankind. As a contemporary example, it would be unwise for a freeway driver to change lanes without first migrating light multiples from the car's rear-view mirror.

There are many scientific and engineering disciplines that use multiples to characterize objects of interest, including the following examples in astronomy, engineering, and seismology.

1) Two of the largest optical telescopes today are the Keck reflecting telescopes in Hawaii, each with a reflecting mirror $10 \mathrm{~m}$ in diameter. Figure 1c depicts one of the Keck mirrors, and the ray diagram illustrates the raypaths for second-order multiples associated with a view of Saturn. Observational astronomers often prefer reflecting telescopes over refraction telescopes because of their relatively lower cost for a given objective diameter, aberration-free imaging, manufacturing simplicity, and compact size.

2) Nondestructive testing of materials uses sonic reverberations to characterize the properties of materials (Prasad and Nair, 2008). An example is the ultrasonic resonance system shown in Figure 1d, in which continuous longitudinal waves are transmitted into the material until, at resonance frequency, standing waves are excited. These standing waves are accompanied by the specimen vibrating at a maximum amplitude. Resonance is detected as a "pip" on the output screen, and the resonance frequency $f^{\text {res }}$ and $\mathrm{P}$-velocity are used to determine the thickness $L=\lambda / 2$ of the specimen.

A related resonance technique, known as the horizontal-to-vertical spectral ratio (HVSR) method (Lane et al., 2008), estimates the resonance frequency of sediment sites from three-component records and relates it to the depth of bedrock. Bonnefoy-Claudet et al. (2006) show that peaks in the HVSR can be linked to the Rayleigh ellipticity peak, the Airy phase of the Love waves, and/or the $\mathrm{SH}$ resonance frequencies, depending on the proportion of these different types of waves in the ambient noise. These parameters give approximately the same value so that the $\mathrm{H} / \mathrm{V}$ peak can sometimes accurately estimate the resonance frequency of the sites. For a two-layer model, the value of $f^{\text {res }}$ is related to the velocity of $\mathrm{S}$-waves $V_{\mathrm{S}}$ and the depth of sediments $L$ :

$$
f^{\mathrm{res}}=\frac{V_{\mathrm{S}}}{4 L} .
$$

It therefore can be used to map the bedrock depth, knowing the $\mathrm{S}$-wave velocity. More generally, the shear velocity as a function of depth can be inverted from the HVSR values as a function of frequency.

3) The modern receiver-function technique images the strong crustal and upper-mantle reflectors from the free-surface reverberations of teleseisms (Kind and Yuan, 2014). It is the most widely used earthquake-imaging technique for delineating reflector boundaries at teleseismic wavelengths. As an example, Figures $2 \mathrm{a}$ and $2 \mathrm{~b}$ depict the raypaths for Pp, PpPmp, Ps, PsPms, and PsPmp arrivals in a crust-mantle model. The

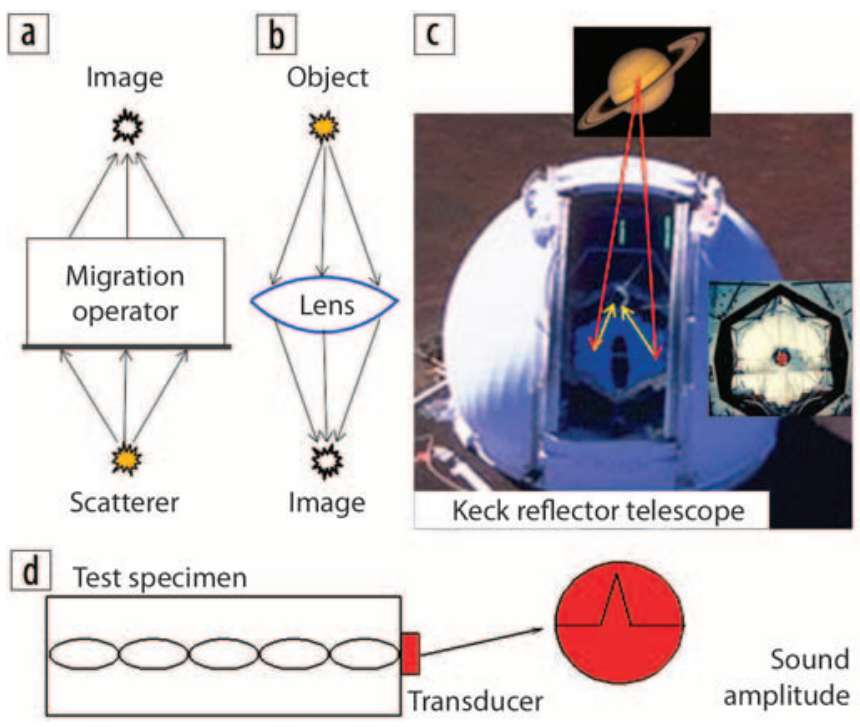

Figure 1. (a) Seismic imaging. (b) Optical imaging. (c) Second-order light multiples (yellow arrows) reflected from the primary mirror (zoomed in the inset on the right) of the Keck reflector telescope. (d) An induced resonance mode in a test specimen of length $L$. 
three-component receivers on the free surface can be rotated to separate $\mathrm{P}$-waves from $\mathrm{S}$ arrivals, and the $\mathrm{Pp}$ arrival at $\mathbf{A}$ can be used to deconvolve the PpPmp arrival at $\mathbf{B}$ to give the receiver function. This receiver function is a trace that contains a virtual Pmp arrival as if it were excited by a virtual source at $\mathbf{A}$ and recorded by a receiver at $\mathbf{B}$. In other words, the free-surface multiple PpPmp is transformed into a virtual Pmp primary.

The receiver function is stacked with other receiver functions that share the same conversion point, and the result is migrated (Chen et al., 2006) to image crustal and uppermantle reflector boundaries. As an example, Figure $2 c$ is the migration image of the Tanlu fault zone computed by migrating $\mathrm{PpPms}$ multiples recorded over the frequency band of 0.03 to $1 \mathrm{~Hz}$. This image reveals fine-scale structures of the crust, Moho, and possibly upper-mantle discontinuities (Chen et al., 2006). A significant benefit of receiver functions is that they can illuminate subsurface areas that are not accessed easily, such as marine environments (Kind and Yuan, 2014).

4) Ambient-noise seismology transforms multiple scattering and reflections in the earth into coherent signals that can be imaged for tectonic structures. For a uniform distribution of noise sources in a heterogeneous nonattenuative earth, the crosscorrelation and stacking of noise records converge to the complete Green's function of the medium, including all reflection, scattering, and propagation modes (Campillo et al., 2014). Ambient-noise seismology and its reuse of noise (multiple scattered arrivals) are considered by many seismologists as a revolutionary advance in earthquake seismology.

As an example, Figure $2 \mathrm{~d}$ depicts the snapshot of a virtual earthquake in the United States. This snapshot was computed by crosscorrelating and stacking several years of noise recorded by USArray data. Inverting the surface waves at low frequencies gives $\mathrm{S}$-wave tomograms at depths (Figure 2e) down to the upper mantle. These virtual data also can be used to reconstruct body-wave reflections from the coremantle boundary (Lin et al., 2013).

It is apparent that many areas of science and engineering use multiple reflections to characterize objects of interest, and thus the explorationist might wonder how multiples can be used to discover hydrocarbons. This article and its companion article in this issue (Guo et al., 2015) present an overview of some important applications of imaging multiple reflections in exploration geophysics. Imaging can be either migration of multiples for the reflectivity distribution or inversion of multiples for the subsurface velocity model.

\section{VSP and OBS mirror migration}

Several applications of mirror migration will be presented for VSP and OBS data. The resulting images will be used to assess the strengths and limitations of mirror migration.

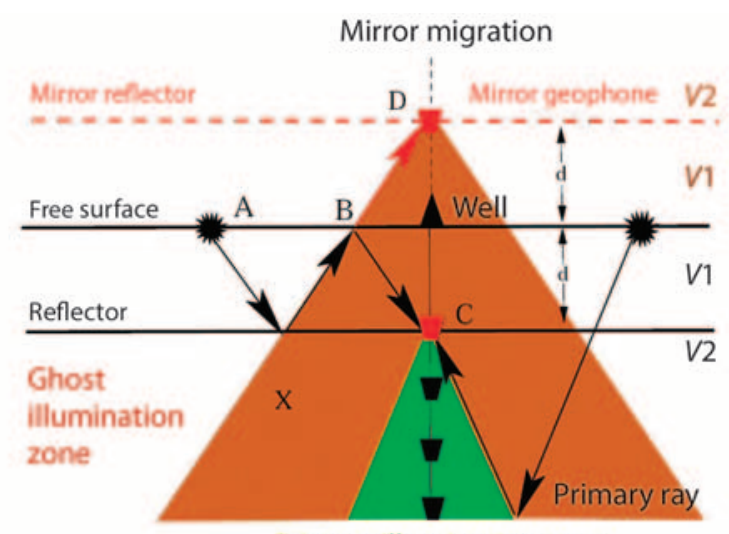

Primary illumination zone

Figure 3. $\mathrm{AXBC}$ is the first-order ghost ray, and $\mathrm{AXBD}$ is the mirror ray. The two-layer medium with velocities $V 1$ and $V 2$ is mirrored above the free surface so that the ghost and mirror reflections have the same traveltimes, $\tau_{\mathrm{AXBD}}=\tau_{\mathrm{AXBC}}$. The mirror ray AXBD is that of a virtual primary recorded by the red receiver at $\mathrm{D}$.

Figure 3 depicts the rays for the first-order multiple reflection $\mathrm{AXBC}$ and the mirror primary reflection AXBD. The medium and the actual receiver at $\mathbf{C}$ are mirrored above the free surface so that $\tau_{\mathrm{AXBD}}=\tau_{\mathrm{AXBC}}$. (Here, a horizontal free surface is assumed, but this procedure can be adjusted [Jiang et al., 2007] for a free surface with variable topography.)

A significant benefit of migrating the first-order multiple is that it has a much wider illumination zone compared with that of the primary. For example, the green triangular zone in Figure 3 represents the area in which primary reflections can be reflected and then recorded at $\mathbf{C}$ for a walkaway VSP survey. This assumes a layered medium with slight vertical variations in velocity. In comparison, the first-order ghost reflections recorded by the geophone at $\mathbf{C}$ for a walkaway VSP survey can illuminate the region in the brown triangular zone. The apex of this brown triangle becomes elevated with deeper geophones so the area illuminated by firstorder free-surface multiples can approach that of a surface-seismic survey for a sufficiently deep geophone array.

However, the fold of the VSP mirror image will be much less than that of a surface-seismic profile (SSP) survey because VSP surveys typically have far fewer receivers. To compensate, VSP surveys ideally should have a much finer shot interval to increase the fold by binning.

Figure 4 shows an example of VSP mirror migration in which an image migrated from VSP multiples is spliced onto 
a vertical slice of a 3D surface-seismic image (Farmani et al., 2012). Although the 3D SSP survey used thousands of shot and receiver positions, the multiple migration image in Figure 4a is resolved better at shallow depths. However, the migration artifacts in the VSP multiple images are higher than in the surface-seismic images. This is mainly because of the small fold in the VSP data and the out-of-plane energy associated with the complex geologic structures.

The VSP mirror image in Line $\mathrm{B}$ (Figure 4c) is polluted with strong artifacts at deep depths and does not compare well with the SSP image. Farmani et al. (2012) conjecture that the complexity of the subsurface model generates strong out-of-plane events that are not accounted for in the 2D migration of VSP multiples. This suggests that a full 3D VSP survey is required to account for out-ofplane effects. For these examples, the usable bandwidth of the VSP multiple migration image was 1.5 times wider than that of the surface-seismic survey (Figures 4e and 4f).

Mirror migration also can be applied to OBS data in which oceanbottom nodes are mirrored above the free surface, as shown in Figure $5 \mathrm{~b}$. Here, first-order multiples are transformed into virtual primaries recorded by virtual OBS nodes mirrored high above the free surface. The height of these virtual nodes is equal to the depth of the seafloor. The benefit is that the illumination area is widened greatly, especially in deep ocean environments.

As an example, Ronen et al. (2012) use mirror migration of OBS data to identify shallow-drilling hazards in the North Sea. Figure $5 \mathrm{~d}$ shows that the OBS mirror-migration image fills in the blind zone (green dashed box) that is not viewable by conventional migration of primaries. In this example, the drilling hazard is the gas zone indicated by bright spots at shallow depths. Ronen et al. (2012, p. 467) conclude that "as expected, the upgoing image (migrated from conventional primary reflections) fails to image the seabed and shallow drilling hazards.”

Another benefit of free-surface multiples is that they provide low-wavenumber information about the model for fullwaveform inversion (FWI). The tomographic operator for FWI resembles an elliptical rabbit ear whose maximum width is approximately proportional to $\sqrt{\lambda L}$, where $L$ is the raypath length. Longer raypaths lead to fatter wavepaths and therefore lower wavenumbers in the reconstructed model (Huang and Schuster, 2014). Reconstructing lower wavenumbers of the velocity model is important for avoiding cycle skipping in FWI. Therefore, free-surface multiples with longer raypaths allow for lower-wavenumber estimates of the model. This is illustrated in Figure 6, where second-order multiples (blue dots) provide a denser distribution of low wavenumbers compared with primaries (red dots).

\section{Summary}

Mirror migration of free-surface multiples in VSP or OBS data can provide much wider subsurface illumination than the migration of primaries recorded by a limited array of downhole or seabed receivers. For a long walkaway experiment, mirrored free-surface reflections illuminate an area almost as large as from a 2D SSP survey. The limitation of mirror migration is that the multiple can spend as much as twice the time in an attenuative medium compared with the corresponding VSP primary. Thus, a mirror-migration image will have greater sensitivity to migration-velocity errors and will have less resolution than the corresponding VSP primary 


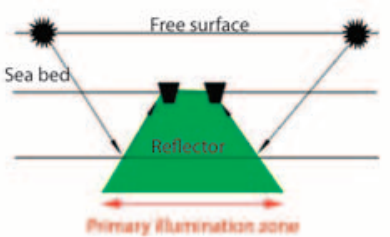

b

First-order OBS ghost illumination

C North Sea: primary migration image $\mathrm{d}$
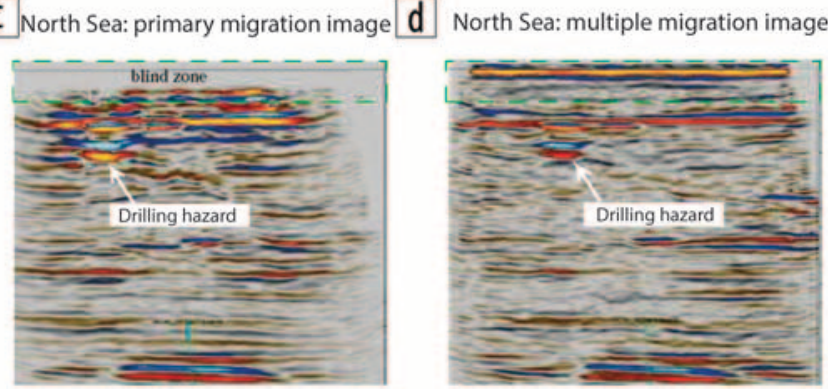

Figure 5. Green illumination zones for OBS (a) primaries and (b) mirrored first-order downgoing ghosts. (c) and (d) Primary and mirror images computed by Ronen et al. (2012).

image. Because of the small number of receivers and sources in a $2 \mathrm{D}$ walkaway VSP survey, any mirror-migration image typically will have more artifacts and less signal-to-noise ratios than the corresponding SSP images. This problem can be mitigated partly by having a finer sampling interval in both the source and receiver distributions. TIE:

Corresponding author: gerard.schuster@kaust.edu.sa

\section{References}

Bonnefoy-Claudet, S., F. Cotton, and P. Y. Bard, 2006, The nature of noise wavefield and its applications for site effects studies: A literature review: Earth-Science Reviews, 79, nos. 3-4, 205227, http://dx.doi.org/10.1016/j.earscirev.2006.07.004.

Campillo, M., P. Roux, and N. Shapiro, 2014, Seismic ambient noise correlation, in H. K. Gupta, ed., Encyclopedia of solid earth geophysics: Encyclopedia of Earth Sciences Series: Springer-Verlag, 1230-1236.

Chen, L., T. Zheng, and W. Xu, 2006, A thinned lithospheric image of the Tanlu fault zone, eastern China: Constructed from wave equation based receiver function migration: Journal of Geophysical Research: Solid Earth: 111, no. B9, http://dx.doi. org/10.1029/2005/JB003974.

Farmani, M. B., B. Atkinson, J. Muzi, N. Brooks, T. Hilton, and J. O'Brien, 2012, Imaging first order multiples in VSP data: A detailed field study: 82nd Annual International Meeting, SEG, Expanded Abstracts, http://dx.doi.org/10.1190/segam2012-0038.1.

Guo, B., J. Yu, Y. Huang, S. M. Hanafy, and G. T. Schuster, 2015, Benefits and limitations of imaging multiples: Interferometric and resonant migration: The Leading Edge, this issue.

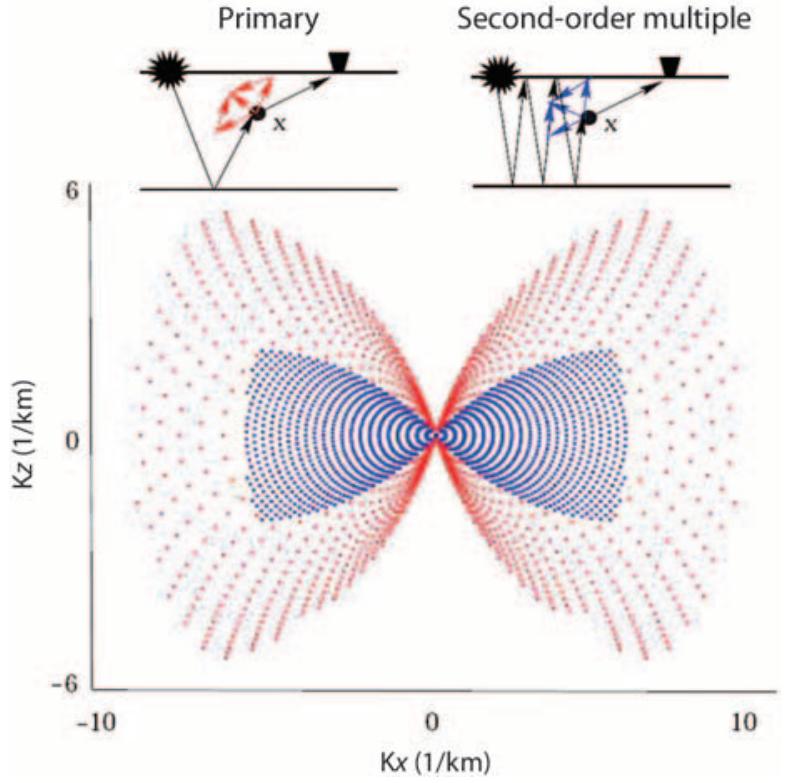

Figure 6. Wavenumber spectra for the tomographic operator associated with primaries (red) and second-order multiples (blue) of a twolayer model.

Huang, Y., and G. T. Schuster, 2014, Resolution limits for wave equation imaging: Journal of Applied Geophysics, 107, 137148, http://dx.doi.org/10.1016/j.jappgeo.2014.05.018.

Jiang, Z., J. Sheng, J. Yu, G. T. Schuster, and B. E. Hornby, 2007, Migration methods for imaging different-order multiples: Geophysical Prospecting, 55, no. 1, 1-19, http://dx.doi. org/10.1111/j.1365-2478.2006.00598.x.

Kind, R., and X. Yuan, 2014, Seismic, receiver function technique, in H. K. Gupta, ed., Encyclopedia of solid earth geophysics: Encyclopedia of Earth Sciences Series: Springer-Verlag, 1258-1269.

Lane, J., E. White, G. Steele, and J. Cannia, 2008, Depth using the horizontal-to-vertical (H/V) ambient-noise seismic method, in Proceedings of Colorado Environmental and Engineering Geophysical Society.

Lin, F.-C., V. C. Tsai, B. Schmandt, Z. Duputel, and Z. Zhan, 2013, Extracting seismic core phases with array interferometry: Geophysical Research Letters, 40, no. 6, 1049-1053, http:// dx.doi.org/10.1002/grl.50237.

Prasad, J., and C. G. Nair, 2008, Non-destructive test and evaluation of materials: Tata McGraw-Hill Education.

Ronen, S., A. Rokkan, R. Bouraly, G. Valsvik, L. Larson, E. Ostensvig, J. Paillet, A. Dynia, A. Matlosz, S. Brown, S. Drummie, J. Holden, K. Koster, D. Monk, and M. Swanson, 2012, Imaging shallow gas drilling hazards under three Forties oil field platforms using ocean-bottom nodes: The Leading Edge, 31, no. 4, 465-469, http://dx.doi.org/10.1190/ tle31040465.1.

Schuster, G. T., 2001, Galileo was a migrationist, in Utah Tomography and Modeling/Migration Development Project, 2001 midyear report, Part II: Migration methods, Report 7: University of Utah, 95-99. 